\title{
ANÁLISIS DEL SISTEMA DE ASEO EN CUANTO AL CUMPLIMIENTO DE LA NORMATIVIDAD AMBIENTAL VIGENTE
}

\section{ANALYSIS OF THE TOILET SYSTEM IN RESPECT OF COMPLIANCE WITH THE ENVIRONMENTAL REGULATION CURRENT}

MSc. Héctor Uriel Rivera Alarcón, Pd.D. Albert Miyer Suarez Castrillon, Ph.D Sir Alexci Suarez Castrillon

${ }^{a}$ Universidad e Pamplona, Programa de Ingeniería Ambiental, Facultad de Ingenierías y Arquitectura, Tel: 5685303 (ext. 140 ) e-mail: hriveraalarcon@gmail.com

${ }^{\mathrm{b}}$ Universidad de Pamplona, Grupo de Investigación Gimup, Ciudad Universitaria, Pamplona Tels: (57+7) 5685303 - 5685304, Colombia, albertmiyer@ unipamplona.edu.co

${ }^{c}$ Universidad Francisco de Paula Santander Ocaña, Grupo de Investigación Grucite, Vía Acolsure Sede el Algodonal, Ocaña - Norte de Santander, Colombia, sasuarezc@ufpso.edu.co

Resumen: El siguiente artículo pretende exponer los datos obtenidos al realizar la evaluación del sistema de aseo en sus cuatro componentes, los cuales son: el barrido y limpieza de áreas públicas, recolección, transporte y disposición final de residuos sólidos urbanos, generados en el municipio de Pamplona departamento de Norte de Santander, el cual vierte sus residuos sólidos municipales en el relleno sanitario regional la Cortada que es administrado por la empresa EMPOPAMPLONA S.A. E.S.P, teniendo en cuenta el cumplimiento de la normatividad ambiental vigente para la prestación del servicio de aseo. Además se presentan alternativas para la el mejoramiento del sistema de aseo dando cumplimiento a la normatividad y para cumplir a cabalidad lo que se encuentra consignado dentro del PGIRS actual.

Palabras clave: Pamplona, normatividad ambiental, sistema de aseo, residuos sólidos, barrido y limpieza de áreas públicas, recolección, transporte, disposición final.

Abstract: The following article aims to present the data obtained when evaluating the toilet system in its four components, which are: sweeping and cleaning of public areas, collection, transportation and disposal of municipal solid waste generated in the municipality of Pamplona department of Norte de Santander, which discharges its municipal solid waste in the Cortada 
regional landfill that is managed by the company EMPOPAMPLONA S.A. E.S.P., taking into account compliance with current environmental regulations for the provision of cleaning services. Also presents alternatives for improving toilet system in compliance with the regulations, and comply fully what is recorded in the current PGIRS.

Keywords: Pamplona, environmental regulations, toilet system, solid waste, sweeping and cleaning of public areas, collection, transportation, disposal.

\section{INTRODUCCIÓN}

La generación de grandes cantidades de residuos sólidos en la población Colombiana, de los cuales su generación es dada por la misma población y el sistema de consumo actual, ha conllevado a la autoridades pertinentes a vigilar, realizar control y seguimientos constantemente a todas la acciones que van encaminadas a la gestión integral de los residuos sólido generados, donde confluyen las sociedad (centros urbanos), de tal manera que dentro del marco de la normatividad ambiental, ha presentado variaciones en sus normas, iniciando con la Ley 99/93 y la Ley 142 de 1994, hasta llegar al punto de fijar unos objetivos para ejecutar la gestión de los residuos sólidos, uno de los cuales, es el mejoramiento de sistemas de eliminación, tratamiento y disposición final de los residuos sólidos; para finalizar emitiendo normas como el decreto 1713 de 2002 y el 838 de 2005, cabe resaltar la importancia que representa el reglamento técnico de agua potable y saneamiento básico, en su título $\mathrm{F}$, (RAS 2000).

Creando de esta manera una serie de prácticas y metodologías que van en beneficio del entorno que nos rodea, minimizando las problemáticas que se puedan ocasionar por no realizar un adecuado manejo de los residuos como: la contaminación del suelo, contaminación de las fuentes hídricas por la infiltración y percolación de lixiviados y contaminación atmosférica con olores y emisión de gases al medio, sin embargo, se ve como se están llevando a cabo estos procesos, que en ocasiones no son los más idóneos, por tal motivo se plantea la evaluación del sistema de aseo en cuatro de sus componentes, en el municipio de Pamplona, Norte de Santander.

\section{MARCO TEÓRICO}

Los residuos sólidos comprenden todos los residuos que provienen de actividades realizadas por el ser humano para satisfacer sus necesidades, que normalmente son sólidos que son desechados como inútiles o superfluos. El termino residuos solido comprende tanto la masa heterogénea de los desechos de la comunidad urbana como la acumulación más homogénea de los residuos agrícolas, industriales y minerales. ${ }^{1}$

El inadecuado manejo de los residuos sólidos contribuye a la contaminación de los suelos y las aguas, al deterioro del paisaje natural y de los centros urbanos, y afecta la salud pública por la proliferación de vectores transmisores de enfermedades. 
El crecimiento urbano y las actividades industriales han aumentado la generación de residuos sólidos, sin desarrollar estrategias para la atención a los problemas ambientales relacionados con la optimización en el uso de los recursos naturales, el desarrollo de programas de seguimiento y control para el cumplimiento de las normas ambientales, y la puesta en marcha de programas de modernización y reconversión industrial orientados a aumentar la eficiencia ambiental y económica.

La apertura económica y los desarrollos institucionales y legales en materia ambiental, han orientado al sector manufacturero nacional hacia una transformación de sus procesos productivos. Estos procesos de modernización y reconversión tecnológica buscan un aumento en la eficiencia económica y ambiental con miras a mejorar la competividad en los mercados nacionales e internacionales.

Para lograr el manejo de los residuos de una forma compatible con el medio ambiente, es fundamental la implementación de una gestión integral de residuos sólidos, que comprende las etapas de reducción en el origen, aprovechamiento, tratamiento, transformación y disposición final controlada. ${ }^{2}$

Dentro de estas actividades encaminadas a el manejo adecuado de los residuos sólidos en nuestro país según el titulo F del ras, la 142/9, el decreto $1713 / 2003$ y complementarios, todo sistema de aseo debes estar integrado por cuatro componentes como son el barrido y limpieza de áreas públicas, recolección, transporte y disposición final de residuos sólidos. Esto con el fin de mitigar y evitar el deterioro del medio, contribuyendo con el cumplimento del art.79 de la constitución política de Colombia.

\section{MATERIALES}

- Información de los procesos del servicio de aseo llevado a cabo por la empresa de Empopamplona.

- Hoja de cálculo para realizar el estudio estadístico.

\section{METODOLOGIA}

Para el análisis del sistema de aseo la metodología empleada se basó principalmente en la observación directa por parte de las personas evaluadoras sobre el cumplimiento en cada uno de los componentes teniendo como referencia lo contenido en la legislación ambiental referente al tema, para que posteriormente se relacionara la información recopilada en unas matrices diseñadas para cada componente en hojas de cálculo, para facilitar su interpretación, estas matrices se basaron en métodos cuantitativos asociados a las variables utilizadas para cada uno de los componentes del sistema de aseo, teniendo en cuentas las características comprendidas en cada componente de acuerdo a la legislación y a ellas según su cumplimiento se les dio un valor porcentual que luego se pasó a una cualificación, para establecer en qué rango se encontraba dicho sistema de aseo.

Tabla 1. Rangos de calificación para el sistema de aseo 


\begin{tabular}{|l|l|}
\hline CALIFICACIÓN & RANGO \\
\hline Excelente & $90-100 \%$ \\
\hline Bueno & $75-89 \%$ \\
\hline Aceptable & $60-74 \%$ \\
\hline Deficiente & $40-59 \%$ \\
\hline Insuficiente & $00-39 \%$ \\
\hline
\end{tabular}

elaboración propia

\section{RESULTADOS Y DISCUSIÓN}

Tabla 2: Resultados del componente de barrido.

\begin{tabular}{|c|c|c|}
\hline \multicolumn{3}{|c|}{ BARRIDO } \\
\hline I & Km sardinel / Mes & $100 \%$ \\
\cline { 2 - 3 } N & Ton / Mes & $100 \%$ \\
\cline { 2 - 3 } F & Diseño y planos (Macro ruta - & $100 \%$ \\
\cline { 2 - 3 } T & Microruta) & \\
\cline { 2 - 3 } E & Horarios y Frecuencias & $100 \%$ \\
\cline { 2 - 3 } C & Registros Estadísticos & $100 \%$ \\
\cline { 2 - 3 } N & Continuidad & $100 \%$ \\
\cline { 2 - 3 } I & Cobertura & $92.82 \%$ \\
\cline { 2 - 3 } C & Plan de Contingencia & $100 \%$ \\
\cline { 2 - 3 } Calidad del Servicio & $80 \%$ \\
\hline P & CUMPLIMIENTO & $\mathbf{9 7 \%}$ \\
E & No Operarios y Cuadrilla Tipo & $100 \%$ \\
\cline { 2 - 3 } R & Herramientas & $60 \%$ \\
\cline { 2 - 3 } S & Pago Parafiscales & $100 \%$ \\
\cline { 2 - 3 } O & Dotación & $100 \%$ \\
\cline { 2 - 3 } N & Elementos Seguridad Industrial & $66 \%$ \\
\cline { 2 - 3 } A & CUMPLIMIENTO & $\mathbf{8 5 \%}$ \\
L & & $\mathbf{9 3 \%}$ \\
\hline & ESTADO DE & \\
\cline { 2 - 3 } & CUMPLIMIENTO &
\end{tabular}

Fuente: elaboración propia

De acuerdo a los datos obtenidos en la tabla No. 1, se tiene que para el componente de barrido en la ciudad de Pamplona, este presenta un cumplimiento del $97 \%$ en cuanto a lo obtenido en la información técnica, y un
$85 \%$ en lo pertinente al personal, concerniente a los empleados que llevan a cabos esta actividad, para un cumplimiento total del componente del $93 \%$, que es un cumplimiento excelente al ser mayor del 90\%; la matriz de evaluación presenta unas casillas que fueron manejadas a criterio del evaluador como calidad, la calificación de la matriz se realizó analizando los aspectos que conforman el componente de barrido, y se aplicó a los componentes restantes.

Tabla 3. Resultados del componente de la recolección.

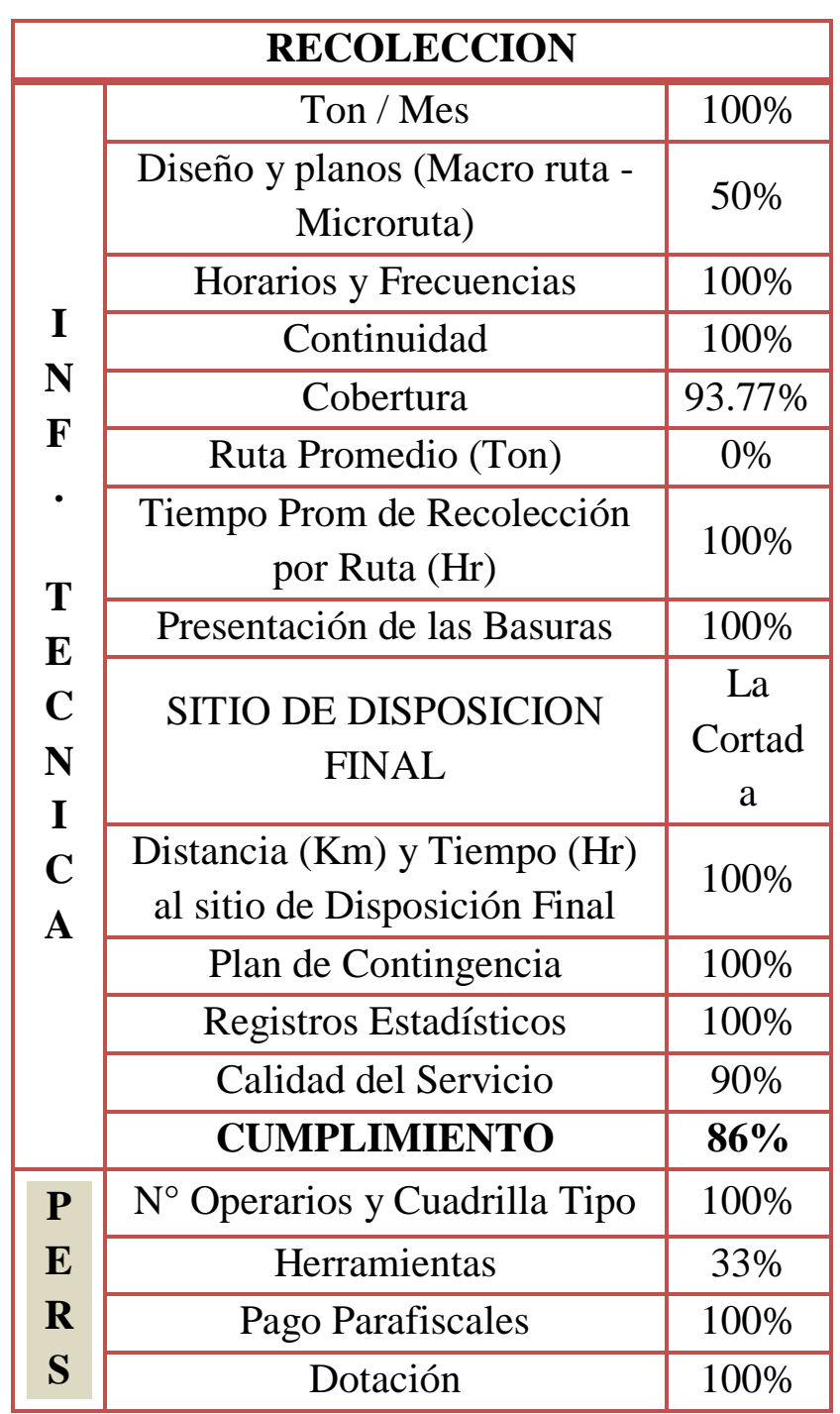




\begin{tabular}{|c|c|c|}
\hline O & Elementos Seguridad Industrial & $33 \%$ \\
\cline { 2 - 3 } A & CUMPLIMIENTO & $\mathbf{7 3 \%}$ \\
L & & \\
\hline & ESTADO DE & $\mathbf{8 2 \%}$ \\
\hline
\end{tabular}

Fuente: elaboración propia

Los resultados conseguidos del componente de recolección, se presentan en la tabla No.2, al igual que la tabla No. 1, se realizó una clasificación de información técnica y personal, para la cual se obtuvieron $86 \%$ y 73 , respectivamente para un estado de cumplimiento de todo el componente del $82 \%$, a pesar de ser un poco menor que el componente anterior, el cumplimiento es bueno.

Tabla 4: Resultados del componente de transporte.

\begin{tabular}{|c|c|c|}
\hline \multicolumn{3}{|c|}{ TRANSPORTE } \\
\hline & Estado de Vehículos & $100 \%$ \\
\cline { 2 - 3 } I & Identificación (color, logos, & $100 \%$ \\
placa) & \\
\cline { 2 - 3 } N & Equipo de Radiocomunicación & $50 \%$ \\
F & y Exostos & \\
\cline { 2 - 3 } : & Caja compactadora Hermética & $100 \%$ \\
\cline { 2 - 3 } T & Equipo de Stand-By & $100 \%$ \\
\cline { 2 - 3 } E & Lavado del equipo & $100 \%$ \\
\cline { 2 - 3 } C & Extintor - Botiquín - Equipo de & $100 \%$ \\
N & Carretera - Estribos & \\
\cline { 2 - 3 } I & Programas de mantenimiento & $100 \%$ \\
\cline { 2 - 3 } C & Hoja de vida de los equipos & $100 \%$ \\
\cline { 2 - 3 } A & Registros estadísticos por & $100 \%$ \\
& equipo & $100 \%$ \\
\hline & CUMPLIMIENTO & $\mathbf{9 5 \%}$ \\
\hline P & No Operarios taller & $100 \%$ \\
\cline { 2 - 3 } E & Herramientas & $100 \%$ \\
\cline { 2 - 3 } R & Pago Parafiscales & $100 \%$ \\
\cline { 2 - 3 } S & Dotación & $75 \%$ \\
\cline { 2 - 3 } & &
\end{tabular}

\begin{tabular}{|c|c|c|}
\hline $\mathbf{O}$ & Elementos Seguridad Industrial & $33 \%$ \\
$\mathbf{N}$ & CUMPLIMIENTO & $\mathbf{8 2 \%}$ \\
$\mathbf{A}$ & & \\
$\mathbf{L}$ & & \\
\hline & ESTADO DE & $\mathbf{9 0 \%}$ \\
& CUMPLIMIENTO & \\
\hline
\end{tabular}

Fuente: elaboración propia

Para el componente de transporte, en el sistema de aseo del municipio de Pamplona, presenta una calificación del 95\% perteneciente a la información técnica y un $82 \%$ para la personal, para un total del $90 \%$ de todo el modulo de transporte, siendo este un resultado más excelente, aunque en cuanto personal es bueno debido a que no cumplen con algunos de los requisitos exigidos por la ley.

Tabla No.5: Resultados del componente de la disposición final.

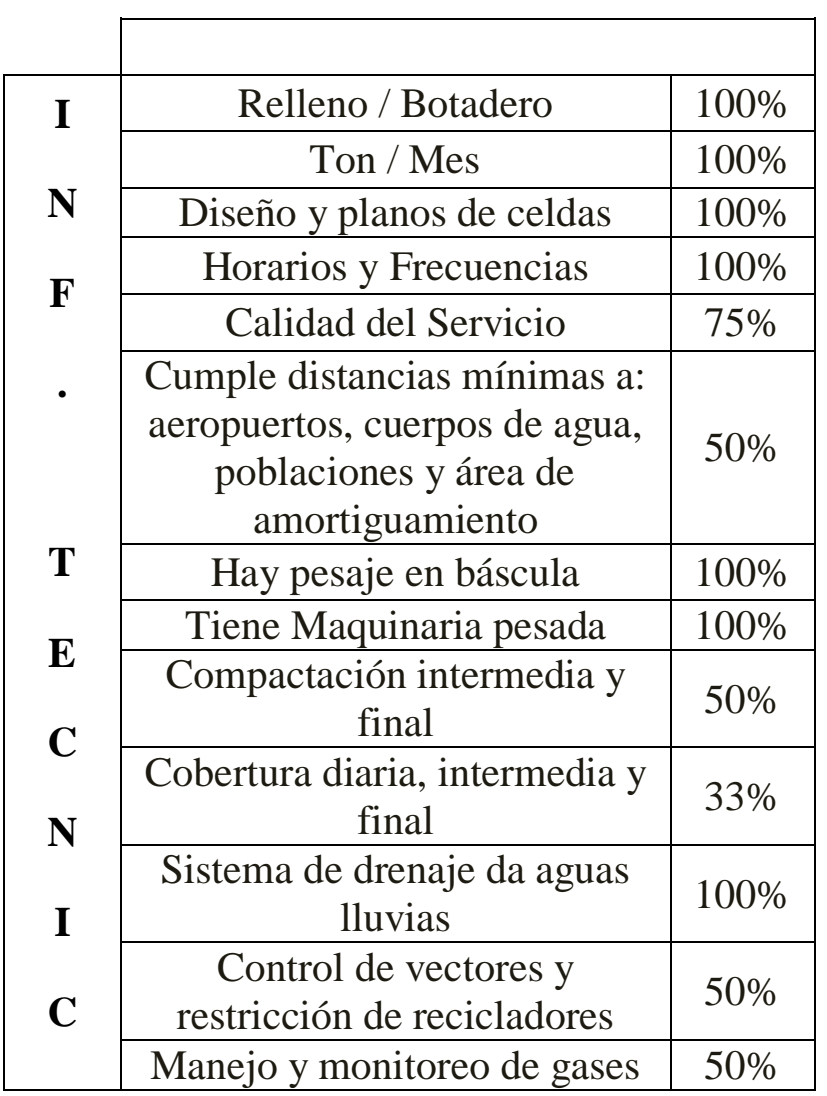




\begin{tabular}{|c|c|c|}
\hline \multirow[t]{7}{*}{$\mathbf{A}$} & $\begin{array}{l}\text { Manejo y monitoreo de } \\
\text { lixiviados }\end{array}$ & $50 \%$ \\
\hline & Manejo paisajístico & $100 \%$ \\
\hline & $\begin{array}{l}\text { Plan de cierre, monitoreo y } \\
\text { seguimiento posterior }\end{array}$ & $100 \%$ \\
\hline & $\begin{array}{c}\text { Hay Reciclaje Técnico } \\
\text { (compost, selección de } \\
\text { residuos) }\end{array}$ & $0 \%$ \\
\hline & Plan de Contingencia & $100 \%$ \\
\hline & Registros Estadísticos & $100 \%$ \\
\hline & CUMPLIMIENTO & $77 \%$ \\
\hline $\mathbf{P}$ & $\mathrm{N}^{\circ}$ Operarios & $100 \%$ \\
\hline \multirow{2}{*}{$\mathbf{E}$} & Herramientas & $50 \%$ \\
\hline & Pago Parafiscales & $100 \%$ \\
\hline $\mathbf{R}$ & Dotación & $100 \%$ \\
\hline $\mathbf{S}$ & $\begin{array}{l}\text { Elementos Seguridad } \\
\text { Industrial }\end{array}$ & $50 \%$ \\
\hline $\mathbf{O}$ & \multirow{4}{*}{ CUMPLIMIENTO } & \multirow{4}{*}{$80 \%$} \\
\hline $\mathbf{N}$ & & \\
\hline $\mathbf{A}$ & & \\
\hline \multirow[t]{2}{*}{$\mathbf{L}$} & & \\
\hline & $\begin{array}{c}\text { ESTADO DE } \\
\text { CUMPLIMIENTO }\end{array}$ & $\mathbf{7 4 \%}$ \\
\hline
\end{tabular}

Fuente: elaboración propia

El ultimo componente del sistema de aseo el cual es el de la disposición final, exhibe resultados de $77 \%$ y $80 \%$, en información técnica y personal respectivamente, para un cumplimiento total del $80 \%$, siendo este el porcentaje más bajo en relación con el resto de componente, esto es debido a que no se realizan ninguna práctica para el aprovechamiento de los residuos sólidos y la permanencia de recuperadores ajenos a la empresa en el relleno sanitario como se indica en el decreto 838 de 2005. Toda la información recopilada fue suministrada por Empopamplona S.A E.S.P, debido a que son aspectos relacionados son el servicio prestado por ellos, estas actividades se desarrollaron en escritorio con personal de la empresa.

Tabla No.6: Calificación total del sistema de aseo del municipio de pamplona

\begin{tabular}{|c|c|c|}
\hline $\begin{array}{c}\text { Component } \\
\text { e }\end{array}$ & $\begin{array}{c}\text { Estado de } \\
\text { cumpliment } \\
\text { o }\end{array}$ & $\begin{array}{c}\text { Cumplimient } \\
\text { o Total del } \\
\text { sistema }\end{array}$ \\
\hline Barrido & $93 \%$ & \multirow{2}{*}{8} \\
\cline { 1 - 2 } Recolección & $82 \%$ & \multirow{2}{*}{$84.75 \%$} \\
\cline { 1 - 2 } Transporte & $90 \%$ & \\
\cline { 1 - 2 } $\begin{array}{c}\text { Disposición } \\
\text { final }\end{array}$ & $74 \%$ & \\
\cline { 1 - 2 } & & \\
\hline
\end{tabular}

Fuente: elaboración propia

El sistema de aseo en el municipio de Pamplona presenta un cumplimiento del $84.75 \%$, siendo este una calificación buena, claro está con sus inconvenientes, como son registros de datos de algunas actividades que no se llevan, otra como el correcto uso de los implementos de seguridad que son suministrados a los trabajadores, la permanencia de personal ajeno a la empresa dentro del relleno sanitario y el ultimo pero más relevante es el no aprovechamiento de los residuos por cualquier tipo de práctica, sin embargo, la ponderación adquirida al sistema de aseo no es buena.

\section{RECOMENDACIONES}

$\checkmark$ Realizar una actualización de los datos para mejorar los registros actuales. 
Actualizar la base de datos de la SUI, pues no cuenta con toda la información requerida ni actualizada.

$\checkmark$ Llevar a cabo una supervisión sobre los trabajadores, para que los empleados den una mejor utilización a las herramientas suministradas.

$\checkmark$ Revisar las macro-micro rutas de recolección de la basura e implementar un sistema de GPS para vigilar que los conductores ejecuten las rutas establecidas, y optimizar los tiempos gastados.

$\checkmark$ Plantear y ejecutar un sistema de aprovechamiento de los residuos sólidos generados ya sea en la fuente o el relleno sanitario. $\checkmark$ Planear fórmulas para sacar las personas ajenas al relleno sanitario sin ir a perjudicar su condición socio económica.

\section{CONCLUSIONES}

La empresa administradora del sistema de aseo del municipio de Pamplona (Empopamplona S.A E.S.P), de acuerdo con la evaluación realizada a las actividades que ejecuta, ostenta una buena planeación y operación, solo tiene algunos inconvenientes que llevando a cabalidad las recomendaciones pueden ser corregidos para que la ponderación en su evaluación aumente, pero en términos generales Empopamplona, efectúa un buen trabajo en el manejo del servicio de aseo en sus cuatro componentes.

\section{REFERENCIAS}

APHA (1995). Standard Methods for the Examination of Water and Wastewater. $19^{\text {th }}$ edition. American Public Health Association, Washington, D.C:

Botero, B.M. y R.P. Thomas. 1987. Biodigestor de bajo costo para la producción de combustible y fertilizante a partir de excretas. Manual para su instalación, operación y utilización. Centro Internacional de Agricultura Tropical. Cali, Colombia.

Bruce, A. Kouzeli-Katsiri y P.J. Newman. Anaerobic digestion of sewage sludge and organicagricultural wastes. Elsevier. New York.

CEMAT. Centro Mesoamericano de Estudios sobre Tecnología Aplicada. 1977. Planta de biogas a pequeña escala de la India. Handbook of Appropriate Technology of the Canadian Munger Fundation. Guatemala, Guatemala.

Ministerio del Medio Ambiente y desarrollo, (2003). Decreto 1713 Por el cual se reglamenta la Ley 142 de 1994, la Ley 632 de 2000 y la Ley 689 de 2001, en relación con la prestación del servicio público de aseo, y el Decreto Ley 2811 de 1974 y la Ley 99 de 1993 en relación con la Gestión Integral de Residuos Sólidos 
Ministerio del Medio Ambiente y desarrollo, (2005). Decreto 838 por el cual se modifica el Decreto 1713 de 2002 sobre disposición final de residuos sólidos y se dictan otras disposiciones.

Gestión Integral de los residuos sólidos, Ministerio de Ambiente y Desarrollo Sostenible. http://www.minambiente.gov.co//contenido/contenido.aspx?catID=1273\&conID=7727

TCHOBANOGLOUS, George. THEISEN, Hilary. VIGIL, Samuel. Gestión Integral de residuos sólidos, Volumen 1, Mc Graw Hill.

SUAREZ GOMEZ, Claudia Inés, Problemática y gestión de residuos sólidos peligroso en Colombia. 